\title{
CORRECTION
}

\section{Correction to: Detection of ionic contaminants in unsaturated soils using time domain reflectometry penetrometer}

\author{
Liangtong Zhan ${ }^{1} \cdot$ Qimeng Guo $^{1} \cdot$ Qingyi Mu ${ }^{2} \odot \cdot$ Yunmin Chen ${ }^{1}$
}

Published online: 21 May 2021

(c) Springer-Verlag GmbH Germany, part of Springer Nature 2021

\section{Correction to: Environmental Earth Sciences (2021) 80:330 https://doi.org/10.1007/s12665-021-09618-2}

Unfortunately, the article was published with a derivation error. The formula (7) in the article should be expressed as follows:

$\mathrm{EC}_{\text {water }}=\frac{\left[\frac{\left(\varepsilon_{\text {soil }}\right)^{k}-\left(\varepsilon_{\mathrm{s}}\right)^{k}}{\left(\varepsilon_{\mathrm{a}}\right)^{k}-\left(\varepsilon_{\mathrm{s}}\right)^{k}}+\frac{\left(\varepsilon_{\mathrm{a}}\right)^{k}-\left(\varepsilon_{\mathrm{w}}\right)^{k}}{\left(\varepsilon_{\mathrm{a}}\right)^{k}-\left(\varepsilon_{\mathrm{s}}\right)^{k}} \cdot\left(a \sqrt{\varepsilon_{\text {soil }}}+b\right)\right]^{h-m}}{\left(a \sqrt{\varepsilon_{\text {soil }}}+b\right)^{h}}\left(\mathrm{EC}_{\text {soil }}-\mathrm{EC}_{\text {surface }}\right)$

We regret the inconvenience caused.

The original article can be found online at https://doi.org/10.1007/ s12665-021-09618-2.

Qingyi $\mathrm{Mu}$

qingyimu@mail.xjtu.edu.cn

Liangtong Zhan

zhanlt@zju.edu.cn

Qimeng Guo

qimengguo@zju.edu.cn

Yunmin Chen

chenyunmin@zju.edu.cn

1 MOE Key Laboratory of Soft Soils and Geoenvironmental Engineering, Zhejiang University, Hangzhou 310058, China

2 Department of Civil Engineering, Xi' an Jiaotong University, Xi'an 710049, China
Publisher's Note Springer Nature remains neutral with regard to jurisdictional claims in published maps and institutional affiliations. 